\title{
Prehospital cardiac arrest in Leicestershire: targeting areas for improvement
}

\author{
T B Hassan, F G Hickey, S Goodacre, G G Bodiwala
}

\begin{abstract}
Objective-To identify the impact of advanced life support skills on outcome for prehospital cardiac arrest in a defined population and to assess the value of certain physiological variables in predicting the outcome in those successfully resuscitated in the accident and emergency (A\&E) department; to identify areas for improvement in the outcome of such patients.
\end{abstract}

Design-Prospective 12 month study. Setting-Leicestershire, United Kingdom. Main outcome measure-Survival to hospital discharge and status at 6 months. Results-266 patients were identified as having suffered a prehospital cardiac arrest; of these, 86 had their resuscitation attempt terminated in the community by a general practitioner and 180 were transferred to the A\&E department of the Leicester Royal Infirmary. Of the latter, 159 were felt to be of cardiac aetiology, and 19 were eventually discharged from hospital. All survivors had experienced a witnessed cardiac arrest, ventricular fibrillation (VF) being identified as the initial rhythm. After adjusting for age and sex using logistic regression, the Glasgow coma score (GCS) was found to be associated with subsequent mortality $\left(\chi^{2}=18 \cdot 22\right.$ on $2 \mathrm{df}, P<0 \cdot 0001$ ). Compared to a baseline GCS of 9-15, the relative odds of death for a GCS of 3 were $25 \cdot 3$ (95\% confidence interval $4 \cdot 3$ to $149 \cdot 9$ ), while a GCS of 4-8 gave a relative odds of death of $12.18(95 \%$ CI 1.8 to $80 \cdot 2$ ). No significant association was found between postarrest arterial $\mathbf{~ p H}$ and mortality.

Department of A\&E Medicine, Leicester Royal Infirmary, Infirmary Square, Infirmary Square, T B Hassan G G Bodiwala

Department of A\&E Medicine, Sligo General Hospital, The Mall, Sligo, Republic of Ireland F G Hickey

Department of A\&E Medicine, St James's University Hospital, Beckett St,

Leeds LS9 7TF

$S$ Goodacre

Correspondence to: T B Hassan.

Accepted for publication 12 January 1996
Conclusions-The immediate GCS on admission is a predictor of outcome and it is important to monitor its trend in the first $24 \mathrm{~h}$. Multidisciplinary audit and joint guidelines with other specialties are important in optimising the care of these patients.

( F Accid Emerg Med 1996;13:251-255)

Key terms: prehospital cardiac arrest; prognostic indicators

Patients suffering a prehospital cardiac arrest are dependent upon a number of time-critical steps in order to produce a return of spontaneous circulation, leading to an increased likelihood of survival. Rapid access to the local emergency medical services (EMS) allied with the use of basic and advanced life support
(BLS, ALS) techniques has led to improved outcomes in a number of EMS systems worldwide. ${ }^{1-7}$ The benefits of advanced life support measures have been studied extensively in the USA. At present defibrillation is the only intervention noted to have a proven significant effect ${ }^{8}$ although the other measures are likely to provide a cumulative effect.

Although Pantridge and Geddes ${ }^{9}$ were the first in the world to show the benefits of identifying and treating life threatening arrhythmias in the prehospital environment, advances in the use of ALS skills in the prehospital management of cardiac arrest have been slow to develop in the United Kingdom. Hampton et $a l^{10}$ in 1978 found no improvement in survival in a randomised trial of prehospital coronary care. Subsequently, however, other studies have shown beneficial outcomes, ${ }^{11-14}$ especially from the successful use of semiadvisory external defibrillators (SEDs).

In Leicestershire, the paramedic programme has continued to develop since 1989 and by the end of 1995 every front line ambulance had a paramedic on board, in line with government policy. In 1991, all front line ambulances were equipped with SEDs. This study aimed to evaluate the effectiveness of the Leicester EMS which included both paramedics and emergency medical technicians trained in defibrillation (EMT/D). The accuracy of certain variables in predicting outcome in those who were successfully resuscitated in the accident and emergency (A\&E) department was also assessed. We hoped that the study would act as a focus to identify areas for improvement in the management of such patients.

\section{Methods}

The Leicester Royal Infirmary is a 1107 bedded teaching hospital with the only major A\&E facility in Leicestershire, serving a population of 900000 . From 1 February 1992 to 31 January 1993, all patients suffering a prehospital cardiac arrest and brought to the Leicester Royal Infirmary A\&E department were included in the study. Patients in whom BLS/ ALS measures were performed but who were declared dead at the scene by local general practitioners and subsequently brought to the Leicester Royal Infirmary were also included in the study in order to conform to the Utstein template for uniform reporting of prehospital cardiac arrest. ${ }^{15}$

Several prehospital cardiac arrests were transferred from the peripheries of the county to smaller A\&E facilities outside Leicestershire (for example, Kettering and Nuneaton). These 
patients were not included in the study and did not come from a clearly defined population that could be excluded from the population denominator served by the EMS. Other exclusions included patients suffering a cardiac arrest due to trauma, drowning, poisoning, and those under 16 years of age. Patients who had suffered a cardiopulmonary arrest secondary to a variety of diagnoses including cerebrovascular accident or pulmonary embolism were included in the non-cardiac subgroup of patients.

The definition and management of prehospital cardiac arrest by the Leicestershire Ambulance and Paramedic Service during the study period was along standard Resuscitation Council guidelines. ${ }^{16}$ The use of semiadvisory external defibrillators by the EMT/D crews is along guidelines described by Cobbe et al. ${ }^{13}$ Most ambulances in Leicestershire are equipped with Laerdal Heartstart 2000 or 3000 series, although several have manual defibrillators. EMT/D crews are advised to transport the patient to the $A \& E$ department once a palpable output has been achieved, or if no shock is indicated but the patient remains in cardiac arrest. Paramedic crews use ALS skills appropriately and ideally transport patients once an output has been achieved or where a resuscitation attempt has been prolonged without success. On certain occasions local general practitioners were available to provide support in the resuscitation attempt or to certify death at the scene. During the study period, the Leicestershire Ambulance and Paramedic Service had approximately 50 paramedic staff who were fully trained in advanced airway control, defibrillation, and drug administration. The manual defibrillators were carried only by paramedic crews.

All patients presenting to the $\mathrm{A} \& \mathrm{E}$ department in cardiac arrest or post-cardiac arrest were prospectively entered into the study by using forms specifically designed for the purposes of the study. Resuscitation was along standard Resuscitation Council (UK) guidelines. Patients successfully discharged from the resuscitation room were followed up to discharge from hospital by review of hospital notes, including time in the intensive care unit, total duration of hospital stay, and neurological status on discharge. Information six months after discharge regarding the patient was gained from the general practitioner over the telephone. The influence of the GCS and immediate post arrest $\mathrm{pH}$ on outcome was assessed by logistic regression allowing for sex and age. Differences in the standards or presence of cardiopulmonary resuscitation were assessed using the $\chi^{2}$ test.

\section{Results}

During this 12 month prospective study, 266 patients were identified as having suffered a prehospital cardiac arrest where BLS and/or ALS measures were employed by the Leicestershire Ambulance and Paramedic Service. Eighty six of these patients had their resuscitation terminated by general practitioners who were on, or were called to, the scene in the community. The mean age of these patients was $67 \cdot 8$ years (40 to 104 ) with a male to female ratio of $66: 20$. Mean ambulance response times for this group was 6.83 $\min (1$ to 20$)$.

The outcome of the 180 patients who were transferred to the A\&E department at the Leicester Royal Infirmary is shown in the figure. The mean age was $66 \cdot 1$ years ( 35 to 89 ), with a male to female ratio of 110:70. Mean ambulance response times in this group were similar, at $6.55 \mathrm{~min}$ (2 to 19). Of a total of 159 patients with cardiac arrest of presumed cardiac aetiology, 54 were successfully resuscitated by either the prehospital care personnel or the $A \& E$ staff, to be admitted to the intensive care unit or coronary care unit.

Of these 54 patients, 23 arrived in the $A \& E$ department with an output following a witnessed ventricular fibrillation (VF) arrest, for which they had received dc cardioversion. Seventeen went on to be discharged from hospital. Two other patients suffered a VF arrest in the ambulance, arriving in the $A \& E$ department within 5 and $7 \mathrm{~min}$ respectively and were defibrillated into a stable rhythm. Both went on to be discharged alive from hospital. Eleven of the 19 patients were alive at six months. Nine were self caring and alert, classed as grade 1 on the overall performance category (OPC) of the Glasgow Pittsburgh scale. ${ }^{17} 18$ The two others achieved grade 3 on the Glasgow Pittsburgh OPC, being conscious but dependent on others for daily support.

In those 21 patients suffering a prehospital cardiac arrest of presumed non-cardiac aetiology, four survived to be discharged from $A \& E$. Two of these patients went on to be discharged from hospital alive, one who had suffered a respiratory arrest secondary to myasthenia gravis, the other due to chronic obstructive airways disease. Both had died when their records were reviewed at six months.

The overall survival to discharge from hospital for patients suffering a witnessed VF arrest in the community (including 45 witnessed cardiac arrests of 61 patients in VF not brought to the A\&E department) was $14 \%$ (17/118). There were no survivors in those groups with end stage myocardial disease or asystole as a presenting syndrome or rhythm.

Table 1 shows the effect of CPR and its quality in the prehospital environment in those patients brought to the $A \& E$ department. Professional CPR was arbitrarily defined as being given by medical, nursing, paramedical, or trained first aid responders, and lay CPR as being given by the lay public. The "professional CPR" group had a higher proportion of survivors than expected and this reached statistical significance $\left(\chi^{2}=6.392\right.$ on $2 \mathrm{df}$, $P=0.041)$. The influence of the immediate Glasgow coma score (table 2) and the immediate postarrest arterial $\mathrm{pH}$ (table 3 ) as prognostic indicators for subsequent mortality was assessed by logistic regression. After adjusting for age and sex, the GCS was found to be significantly associated with subsequent mortality $\left(\chi^{2}=18.22\right.$ on $\left.2 \mathrm{df}, P<0.0001\right)$. 


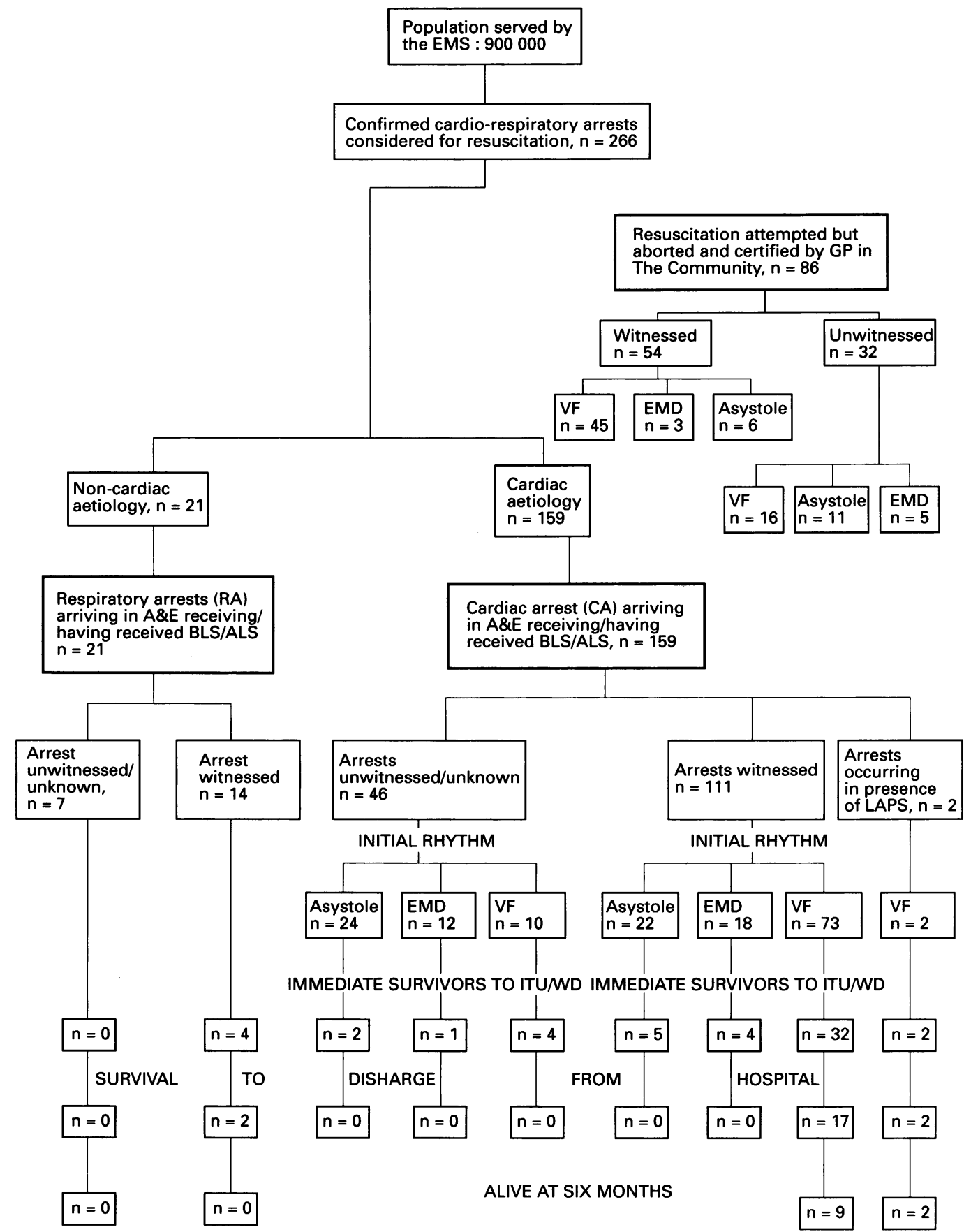

Outcome of 180 patients transferred to the accident and emergency department at the Leicester Royal Infirmary.

Compared to a baseline GCS of 9-15, the relative odds of death for a GCS of 3 were $25 \cdot 3$ ( $95 \%$ confidence interval $4 \cdot 3$ to $149 \cdot 9$ ), while a GCS of 4-8 gave a relative odds of death of $12 \cdot 18(95 \%$ confidence interval $1 \cdot 8$ to $80 \cdot 2)$. The number of patients with results available

Table 1 Influence of bystander cardiopulmonary resuscitation (CPR) in patients suffering a prehospital cardiac arrest

\begin{tabular}{lll}
\hline & $\begin{array}{l}\text { Immediate survivors of } \\
\text { cardiac arrest leaving } \\
\text { the AE } E \text { dept }\end{array}$ & $\begin{array}{l}\text { Unsuccessful } \\
\text { resuscitation in } \\
\text { the AE E dept }\end{array}$ \\
\hline Professional CPR $^{1}$ & 20 & 28 \\
Lay CPR & 3 & 16 \\
None & 17 & 55 \\
Not specified & 14 & 27
\end{tabular}

${ }^{1}$ CPR given by medical, nursing, paramedical or trained first

${ }^{2}$ aid responders. for analysing postarrest arterial $\mathrm{pH}$ and mortality was too small to attain statistical significance. However, a trend towards survival is shown for those with an initial postarrest arterial $\mathrm{pH}$ of $>7 \cdot 2$.

\section{Discussion}

The development of paramedics in the ambulance service and deployment of semiadvisory

Table 2 The immediate post cardiac arrest Glasgow coma score (GCS) as a prognostic indicator of survival

\begin{tabular}{lcc}
\hline GCS & Died & Survived \\
\hline $13-15$ & 0 & 11 \\
$9-12$ & 5 & 2 \\
$4-8$ & 8 & 5 \\
3 & 20 & 3 \\
Total & 33 & 21 \\
\hline
\end{tabular}


Table 3 The immediate post cardiac arrest arterial $\mathrm{pH}$ as a prognostic indicator of survival

\begin{tabular}{lcc}
\hline$p H$ & Died & Survived \\
\hline$>7 \cdot 2$ & 11 & 6 \\
$7 \cdot 0-7 \cdot 19$ & 11 & 3 \\
$<7 \cdot 0$ & 5 & 1 \\
Not stated & 6 & 11 \\
Total & 33 & 21 \\
\hline
\end{tabular}

external defibrillators to every front line ambulance has proved a significant change in the way prehospital cardiac arrest is managed in the United Kingdom. In Leicestershire, this prospective study has shown a discharge rate from hospital of $14 \%$ for patients suffering a witnessed VF arrest in the community. In this series, this includes 86 patients in whom resuscitation was terminated by general practitioners in the community. Others report discharge rates varying from $3 \%$ to $33 \%$. ${ }^{19}$ This variation is due in part to inaccuracies in the exact definition of the denominator which is used for cardiac arrests within an emergency medical service system, making comparisons difficult. ${ }^{20}$ Adherence to the guidelines of the Utstein template should improve the uniformity of such reporting. However, data capture for such reporting styles is difficult in large systems, especially where several hospitals accept these critically ill patients. Gennis et $a l^{21}$ in New York City, developed a concurrent and interactive data collection system for 2329 cardiac arrest patients in a six month period. They concluded that although the method was costly it yielded a highly effective form of data acquisition. Our study, which was the first stage of an ongoing programme of prehospital cardiac arrest audit, employed a system of continuous prospective data collection and updating. Such audit allows trends to be identified within the system and hence can target areas for continuing improvement.

The mean response time by the Leicestershire ambulance and Paramedic Service to a collapsed patient in the study was six minutes. This critical step in minimising the time to providing ALS measures (especially defibrillation) is one of the most important factors influencing outcome. The development of criteria (or priority) based dispatch in the United States has allowed better utilisation of paramedics and emergency technicians for such critically ill patients ${ }^{22}{ }^{23}$ and decreased this response time. It seems likely that criteria based dispatch will be introduced widely in the United Kingdom and audit systems must be in place to assess its effect.

The incidence of bystander CPR in our study was poor, with only a third of patients brought to the $A \& E$ department having being given any form of CPR. Although "professional CPR" was arbitrarily defined, table 1 shows that it was more likely to be associated with a successful outcome. Its influence in affecting the eventual outcome is shown from studies, especially in Seattle, confirming its usefulness as a holding measure until definitive treatment is made available. ${ }^{24}{ }^{25}$ Better teaching programmes in BLS need to be developed in Leicestershire to strengthen this essential link in the chain of survival.

The clear benefit of prehospital defibrillation is confirmed, with 17 of the 21 patients discharged from hospital having required defibrillation as the only intervention in the community. None of the patients requiring other advanced life support procedures (intubation or resuscitation drugs) was alive at six months. This again supports data showing that such measures in themselves have not yet been proven to have a significant impact on eventual outcome in prehospital cardiac arrest. ${ }^{8}$ There were only two patients who arrived in the $A \& E$ department in cardiac arrest who were successfully resuscitated and discharged from hospital neurologically intact. Both had VF as their initial rhythm, occurring in the ambulance on their way to a hospital. This confirms the dismal results of other studies on the poor outcome of patients suffering a prehospital cardiac arrest who are transported to hospital with ongoing resuscitation despite ALS measures. ${ }^{26}{ }^{27}$ Pepe et $a l^{28}$ noted that 24 of the 193 survivors out of 2404 patients with prehospital cardiac arrest had asystole or end stage myocardial disease as their primary problem and this was therefore good evidence that such patients should have aggressive initial resuscitation attempts. These results come from an established EMS system with strong medical involvement and control. Such systems allow better quality assurance programmes to be developed and hence ensure high standards of performance in the field. ${ }^{29}$

In the USA, several states have standing orders to allow resuscitation of cardiac arrests to be terminated in the field after discussion with a doctor in the emergency department. Others have proven that distinct protocols can be drawn up to allow paramedics to declare death at the scene themselves. ${ }^{30}$ In the United Kingdom, ambulance services will either transfer patients to hospital in cardiac arrest with ongoing resuscitation, patients will be declared dead at the scene by the general practitioner, or, in certain areas, mobile medical teams from the local $A \& E$ departments will resuscitate patients and certify them if necessary at the scene. In our study, almost one third of patients were certified at the scene by a local general practitioner, a proportion of whom were actively involved in resuscitation. This may well be appropriate, although a number of factors should be considered. The exposure of a general practitioner to a cardiac arrest situation on an annual basis is low. Training in ALS procedures for general practitioners is variable and hence their confidence and expertise in managing such an event is also likely to be low. Anecdotal discussion with our prehospital personnel suggests that on occasions they have been directed to deviate markedly from standard Resuscitation Council guidelines. Alternatives include better education for general practitioners or a direct communication link with the local $A \& E$ facility and discussion with a senior clinician in the department. The American College of emergency Physicians in a policy statement defined the 
role and responsibility of these "intervener" physicians. ${ }^{31}$ This aspect of prehospital cardiac arrest management in Leicestershire merits further study.

Patients who are successfully resuscitated after a prehospital cardiac arrest are still critically ill and need to be optimally managed in a high dependency or intensive care unit. However, some with end stage disease processes may not merit such aggressive treatment, such decisions ideally being taken jointly by senior clinicians in the $A \& E$ department, intensive care unit, and general medicine. Anecdotal reports of early decision making in the resuscitation room based chiefly on the post arrest GCS led to us assessing this variable as well as the arterial blood $\mathrm{pH}$ in predicting outcome. The GCS proved to be useful in predicting outcome, although, more importantly, assessing the trend in the GCS is highlighted in particular by the three patients who presented with an admission GCS of 3 and went on to be discharged from hospital. This confirms the need to treat such patients aggressively for 24 hours before attempting to assess the degree of hypoxic cerebral insult. ${ }^{32}$

The changes in the management of prehospital cardiac arrest in the United Kingdom have resulted in a significant impact on intensive care unit facilities, as confirmed elsewhere. ${ }^{33} \mathrm{~A}$ number of these patients, having suffered irreversible hypoxic cerebral injury, will end up dying on the intensive care unit after a period of ventilatory and inotropic support. It is important therefore to develop joint guidelines with intensive care unit staff in the assessment and early postresuscitation management of these patients, in order that patients are given the best possible opportunity for survival balanced against often scarce intensive care facilities.

In summary, in Leicestershire the outcome of patients suffering a witnessed VF arrest is equivalent to most other EMS systems in the world apart from that of Seattle and King County. Areas for improvement in the future will concentrate on enhancement of BLS schemes for the lay public within the county, continued development of quality assurance programmes linked to audit, and continuing education for prehospital and in-hospital personnel. The institution of joint guidelines with other specialities involved in the early postresuscitation care of these patients is also essential.

We thank Dr Carol Jagger for her support with the statistical analysis, Mrs Maria Goldsmith (departmental audit assistant) and all the paramedics, emergency technicians, nurses, and doctors who helped to ensure the quality of the data.

1 Eisenberg M, Bergner L, Hallstrom A. Paramedic programs and out-of-hospital cardiac arrest. I. Factors associated with successful resuscitation. Am $\mathcal{F}$ Public Health 1979; 69:30-8.

2 Myerburg RJ, Conde CA, Sung RJ, et al. Clinical, electrophysiologic and hemodynamic profile of patients resuscitated from prehospital cardiac arrest. $\mathrm{Am} \mathcal{f} \mathrm{Med}$ 1980;68:568-76.
3 Cummins RO, Eisenberg MS, Hallstrom AP, Litwin PE. Survival of out-of hospital cardiac arrest with early initiation of cardiopulmonary resuscitation. Am $₹$ Emerg Med 1985;3:114-9.

4 Einarsson O, Jacobsson F, Sigurdsson G. Advanced cardiac life support in the prehospital setting: the Reykjavik experience. F Intern Med 1989;225:129-35.

5 Eisenberg MS, Hadas E, Nuri I, et al. Sudden cardiac arrest in Israel: factors associated with successful resuscitation. Am $\mathcal{F}$ Emerg Med 1988;6:319-23.

6 Vertesi L. The paramedic ambulance: a Canadian experience. Can Med Assoc f 1978;119:25-9.

7 Sammel NL, Taylor K, Selig M, et al. New South Wales intensive care ambulance system: outcome of patients with ventricular fibrillation. Med $\mathcal{F}$ A ust 1981;2:246-50.

8 Pepe PE, Abramson NS, Brown CG. ACLS - does it really work? Ann Emerg Med 1994;23:1037-41.

9 Pantridge JF, Geddes JS. A mobile intensive care unit in the management of myocardial infarction. Lancet 1967;ii: manage

10 Hampton JR, Nicholas C. Randomised trial of a mobile coronary care unit for emergency calls. $B M \mathcal{F} 1978 ; \mathrm{i}$ : coronary

11 Gray AJ, Redmond AD, Martin M. Use of the automatic external defibrillator-pacemaker by ambulance personnel: the Stockport experience. $B M \mathscr{F}$ 1987;294:1133-5.

12 Rowley JM, Mounser P, Garner C, Hampton JR. Advanced training for ambulance crews: implications from 403 consecutive patients with cardiac arrest managed by crews with simple training. $B M \mathcal{F}$ 1987;295:1387-9.

13 Cobbe SM, Redmond MJ, Watson JM, Hollingworth J, Carrington DJ. "Heartstart Scotland" - initial experience of a national scheme for out of hospital defibrillation. $B M \mp 1991 ; 302: 1517-20$.

14 Sedgewick ML, Dalziel K, Watson J, Carrington DJ, Cobbe SM. Performance of an established system of first responder out-of-hospital defibrillation. The results of the second year of the Heartstart Scotland Project in the Utstein Style. Resuscitation 1993;24:75-88.

15 Cummins RO, Chamberlain DA, Abramson NS, et al. Recommended guidelines for uniform reporting of data from out-of-hospital cardiac arrest. The Utstein Style. from out-of-hospital cardiac
Resuscitation 1991;22:1-22

16 Evans TR, ed. $A B C$ of resuscitation, 2 nd ed. London: $B M \mathcal{F}$ Publishing Group, 1990.

17 Jennett $B$, Bond $M$. Assessment of outcome after severe brain damage: a practical scale. Lancet 1975;i:480-4.

18 Brain Resuscitation Clinical Trial I Study Group. A randomized clinical study of cardiopulmonary cerebral resuscitation: design, methods, and patient characteristics. Am $\mathcal{F}$ Emerg Med 1986;4:72-86.

19 Eisenberg MS, Horwood BT, Cummins RO, ReynoldsHaertie R, Hearne TR. Cardiac arrest and resuscitation: a tale of 29 cities. Ann Emerg Med 1990;19:179-86.

20 Eisenberg MS, Cummins RO, Larsen MP. Numerators, denominators, and survival rates: reporting survival from out of hospital cardiac arrest. Am f Emerg Med 1991; 9:544-6.

21 Gennis P, Lombardi G, Gallagher EJ. Methodology for data collection to study prehospital cardiac arrest in New York Collection to study prehospital cardiac arrest in New York 24:194-201.

22 Slovis C, Carruth T, Seitz W, et al. A priority dispatch system for emergency medical services. Ann Emerg Med system for emerge

23 Clawson JJ. Emergency medical dispatch. In: Kuehl AE, ed. EMS medical director's handbook. St Louis: CV Mosby, 1989:59-90

24 Ritter G, Wolfe RA, Goldstein S. The effect of by-stander CPR on survival of out-of-hospital cardiac arrest. $A m$ Heart $\mathcal{F} 1985 ; 110: 932-37$.

25 Eisenberg MS, Bergner L, Hallstrom A. Cardiac resuscitation in the community: importance of rapid provision and implications for program planning. $¥ A M A$ 1979;241:1905-7.

26 Gray WA, Capone RJ, Most AS. Unsuccessful medical resuscitation - are continued efforts in the emergency department justified? $N$ Engl $₹$ Med 1991;325:1393-8.

27 Kellermann AL, Hackman BB, Somes G. Predicting the outcome of unsuccessful prehospital advanced life outcome of unsuccessful prehosp

28 Pepe PE, Levine RL, Fromm RE, et al. Cardiac arrest presenting with rhythms other than ventricular presenting with rhythms other than ventricular fibrillation: contribution of resuscitative efforts to
total survivorship. Crit Care Med 1993;21:1838-43.

29 Holroyd BR, Knopp R, Kallsen G. Medical control: quality assurance in prehospital care. $¥ A M A 1986 ; 256: 1027-31$.

30 Bonnin MJ, Pepe PE, Kimball KT, Clark PS. Distinct criteria for termination of resuscitation in the out of hospital setting. $\mathcal{F} A M A$ 1993;270:1457-62.

31 American College of Emergency Physicians. Direction of prehospital care at the scene of medical emergencies. Ann Emerg Med 1993;23:1145-6.

32 Resuscitation Council (UK). Advanced life support manual. London: Resuscitation Council, 1992

33 Hamer DW, Gordon MWG, Cusack S, Robertson CE. Survival from cardiac arrest in accident and emergency department: the impact of out of hospital advisory defibrillation. Resuscitation 1993;26:31-6. 